\title{
Originalarbeiten
}

\section{Verbleib von Spurenschadstoffen bei der Methylesterherstellung aus Altspeisefett im Technikumsmaßstab}

\author{
Oliver Falk ${ }^{2}$, Hubertus Wichmann ${ }^{1 *}$, Petra Jopke1, Christiane Schmidt-Nädler ${ }^{1}$, Birthe Matthies ${ }^{1}$, Müfit Bahadir ${ }^{1}$ \\ und Roland Meyer-Pittroff ${ }^{2}$
}

${ }^{1}$ Institut für Ökologische Chemie und Abfallanalytik, TU Braunschweig, Hagenring 30, D-38106 Braunschweig

${ }^{2}$ Lehrstuhl für Energie- und Umwelttechnik der Lebensmittelindustrie, TU München, Weihenstephaner Steig 22, D-85350 Freising-Weihenstephan

* Korrespondenzautor (hu.wichmann@tu-bs.de)

DOI: http://dx.doi.org/10.1065/uwsf2004.02.077

\section{Zusammenfassung}

Hintergrund und Ziel. In Deutschland werden jährlich ca. $120.000 \mathrm{t}$ Altspeisefette aus Gastronomie und Lebensmittelindustrie gesammelt. Bis vor kurzem fanden diese Fette größtenteils als Futtermittelbestandteil Verwendung. Ausgelöst durch Futtermittelskandale und die BSE-Krise werden die Fette kaum noch als Tierfutterergänzung eingesetzt. Heute substituieren sie in der chemischen Industrie z.T. frische Pflanzenöle und werden auch als Rohstoff für die Biodieselherstellung verwendet. Diese Randbedingungen legen es nahe, nach neuen Verwertungswegen zu suchen. Von der Deutschen Bundesstiftung Umwelt (DBU) wird daher ein Verbundprojekt finanziert, in dessen Rahmen durch Umesterung der Fette Grundöle für Kühlschmierstoffe (KSS) hergestellt und die KSS-Produkte anschließend in der spanenden Bearbeitung von Metallen in der Praxis getestet werden.

Methoden. In einem ersten Schritt wurden durch analytische Untersuchungen die Eigenschaften und die Qualität von sieben verschiedenen Altspeisefetten festgestellt. Die fettspezifischen Parameter umfassten Gesamtverschmutzung, Sulfatasche, Wassergehalt, Peroxidzahl, Jodzahl, kinematische Viskosität, Säurezahl (freie Fettsäuren) und Fettsäurespektrum.

Im folgenden Schritt wurde eine Verfahrensentwicklung/-optimierung für die Methylesterherstellung unter Einsatz des Rohstoffs Altspeisefett durchgeführt, die in der Konstruktion einer Technikumsanlage mündete. Um den Verbleib von Schadstoffen während der Altfettmethylesterherstellung zu untersuchen, wurden Proben eines der Fette gezielt mit PCB, PAK, PCDD, $\mathrm{PCDF}$ und den Elementen $\mathrm{Al}, \mathrm{Cd}, \mathrm{Cr}, \mathrm{Cu}, \mathrm{Ni}, \mathrm{P}, \mathrm{Pb}, \mathrm{Sn}$ und $\mathrm{Zn}$ versetzt. Diese dotierten Fettproben wurden unter Nachstellung des Verfahrens zur Methylesterherstellung im Labormaßstab umgesetzt und die einzelnen Haupt- und Nebenprodukte anschließend analysiert.

Ergebnisse. Die fettspezifischen Parameter lieferten wertvolle Hinweise für die Auslegung des technischen Prozesses zur Herstellung der Fettsäuremethylester. So erwiesen sich Filtration und Entwässerung der Fette als notwendig. Die für die Kühlschmierstoffherstellung interessierenden gesättigten Fettsäuren repräsentierten $11,3 \%$ bis $31,6 \%$ des Fettsäurespektrums. Da die Mischfettproben einen relativ geringen Anteil an freien Fettsäuren aufwiesen, konnte bei der Entwicklung des Verfahrens zur Methylesterherstellung auf eine basisch katalysierte Umesterung ohne Vorveresterung der freien Fettsäuren zugegriffen werden.
Spurenanalytische Untersuchungen bezüglich anorganischer und organischer Schadstoffe belegten die niedrige Grundbelastung eines Altspeisemischfettes. Dotierexperimente zur Fettsäuremethylester-Herstellung zeigten eine bevorzugte Anreicherung der Schwermetalle im Glycerin. Die organischen Schadstoffe wurden hingegen zu 80 bis $95 \%$ in der Methylesterfraktion nachgewiesen.

Ausblick. In einem nächsten Schritt soll der Prozess der Weiterverarbeitung der Methylester zu Monoalkylestern mit Alkoholen der Kettenlänge C2 bis C8 vorgestellt werden. Damit verbunden werden Ergebnisse chemisch-analytischer Untersuchungen präsentiert werden, die sowohl beim Herstellungsprozess als auch während des Kühlschmierstoffeinsatzes an der Werkzeugmaschine durchgeführt wurden.

Schlagwörter: Altspeisefett; Biodiesel; Fettanalytik; Fettsäuremethylester; Kühlschmierstoff; PAK; PCB; PCDD/F; Schwermetalle

\section{Abstract}

Fate of Trace Pollutants During the Production of Methylesters Based on Waste-edible Fats

Aims and Scope. In Germany, 120,000 tons per year of waste edible fats are collected from the catering and the food industry. Until recently, these fats have widely been used as a nutritional additive for poultry and other animals fodder. Due to the BSE crisis and some affairs based on dioxins in feeding stuff, waste fats are now barely used as fodder. Currently, these fats substitute fresh vegetable oils in the chemical industry and are used as raw material for the production of biodiesel.

Therefore, alternative fields of application are required. In this context, the Deutsche Bundesstiftung Umwelt (DBU) is sponsoring a joint research project which deals with the production and testing of cooling lubricants based on monoesters made from waste edible fats.

Methods. In a first step, characteristics and quality of wasteedible fats of different origins were chemically analysed and monitored. The investigations covered the following fat specific parameters: total contamination, sulphate ash, water content, peroxide number, iodine value, kinematical viscosity, neutralisation number (free fatty acids) and fatty acid spectra.

In the next step, a process development/optimisation was carried out for the production of methylesters based on the raw material waste fat, leading to the construction of a pilot plant. To investigate the fate of trace pollutants during the production process of waste-fat methylester, samples were systematically 
contaminated with polycyclic aromatic hydrocarbons, polychlorinated biphenyls, polychlorinated dibenzo-p-dioxins, polychlorinated dibenzofurans and the elements $\mathrm{Al}, \mathrm{Cd}, \mathrm{Cr}, \mathrm{Cu}$, $\mathrm{Ni}, \mathrm{P}, \mathrm{Pb}, \mathrm{Sn}$, and $\mathrm{Zn}$. These contaminated fat samples were transesterified in laboratory scale. The primary and by-products were analysed subsequently.

Results. Valuable hints on the design of the technical process of fatty acid methylester production based on waste edible fats were gained by regarding the fat specific parameters. For example, filtration and dewatering of the waste fats proved necessary. The saturated fatty acids, most wanted for the production of cooling lubricants due to their high oxidation stability, were present in the range of $11,3 \%$ to $31,6 \%$. Due to the low content of free fatty acids, a base catalysed process occurred more suitable for the transesterification of the waste edible fats.

Trace analytical investigations concerning inorganic and organic pollutants proved a low basic contamination of the waste edible fats. Experiments with systematic contamination of the fats indicated an accumulation of the heavy metals in the glycerol phase during the transesterification process, whereas the organic pollutants were detected in the methylester fraction by amounts of $80 \%$ to $95 \%$.

Outlook. In a next step, the further processing of the methylesters to monoesters with alcohols of the chain length $\mathrm{C} 2$ to $\mathrm{C} 8$ will be presented. Associated results of chemical-analytical investigations on the process and the application of the cooling lubricants will also be given.

Keywords: Biodiesel; cooling lubricant; fat analysis; fatty acid methylester; heavy metal; PAH; PCB; PCDD/F; waste edible fat

\section{Einleitung}

Unter Altspeisefett versteht man gebrauchtes, evtl. verunreinigtes, überlagertes oder verdorbenes Frittier-, Brat-, Back-, Grill- und Speisefett, das auf Grund von veränderten Eigenschaften nicht mehr für den menschlichen Verzehr bzw. zum bestimmungsgemäßen Gebrauch verwendet werden kann [1]. Genaue Zahlen über die in Deutschland gesammelten Mengen an Altspeisefett aus Gastronomie und Lebensmittelindustrie sind auf Grund der Vielzahl von kleinen AltfettSammelbetrieben schwer zu ermitteln. Die Niederrheinische Fettschmelze GmbH ist nach eigenen Angaben mit $20.000 \mathrm{t} / \mathrm{a}$ der größte Altfettentsorger in Deutschland. Die Firma Lesch ist in Bayern und Baden-Württemberg der größte Altfettentsorger mit derzeit $12.000 \mathrm{t} / \mathrm{a}$ [2]. Laut Schätzungen liegt das Sammelpotential an Brat- und Frittierfetten aus Gastronomie und Lebensmittelindustrie in Deutschland zwischen 150000 und $380.000 \mathrm{t} / \mathrm{a}[3,4]$. Die tatsächlich gesammelte Menge beträgt etwa 100.000 bis 120.000 t/a [1].

Die Zwischenfälle der letzten Jahre (z.B. Dioxin- und PCBSkandal in Belgien; Mineralöl im Hühnerei; Klärschlamm, Hydraulik- und Transformatorenöl, Wachstumsbeschleuniger und Antibiotika im Tierfutter) verstärken das Bestreben, auch leicht kontaminierbare Altspeiseöle und Altfette aus der Futtermittelproduktion zu entfernen, da Schadstoffe über die Tierverfütterung in die menschliche Nahrung gelangen können [5].

In diesem Zusammenhang wird in einem von der Deutschen Bundesstiftung Umwelt (DBU) geförderten Forschungsvorhaben die Verwertung von Altspeisefetten und technischen tierischen Fetten als Rohstoff für die Produktion von Kühlschmierstoffen (KSS) untersucht (DBU, Az. 17205).
Hierzu wurden verschiedene Monoalkylester auf Basis von Altspeisefetten hergestellt, die anschließend am Institut für Werkzeugmaschinen und Fertigungstechnik, TU Braunschweig, auf ihre technologische Eignung im Zerspanungsprozess getestet wurden. Neben der Bearbeitung wirtschaftlicher, technologischer und ökologischer Fragestellungen, mußten rückstandsanalytische Untersuchungen zur Charakterisierung der Rohstoffe sowie zu den Aspekten Arbeitsplatzsicherheit und Produktakzeptanz durchgeführt werden.

Ziel der Untersuchungen war es zunächst, Eigenschaften un'd Qualität des Rohmaterials Altspeisefett durch die Bestimmung von fettspezifischen Parametern und im Fall eines Mischfettes durch organische und anorganische Spurenanalytik festzustellen. Um den Verbleib von Schadstoffen während der Methylesterherstellung zu untersuchen, wurde eines der Fette gezielt mit PCDD, PCDF, PCB, PAK und den Elementen $\mathrm{Al}, \mathrm{Cd}, \mathrm{Cr}, \mathrm{Cu}, \mathrm{Ni}, \mathrm{P}, \mathrm{Pb}, \mathrm{S}, \mathrm{Sn}$ und $\mathrm{Zn}$ versetzt. Das Verfahren zur Methylesterherstellung wurde hierfür im Labormaßstab nachgestellt.

\section{Material und Methoden}

\subsection{Probenmaterial}

Es wurden sieben verschiedene Altspeisefettproben von einem Altfettverwerter bezogen. Die Proben wurden direkt aus den Sammeltonnen bzw. nach der Reinigung mittels mechanischer Dekantierung aus der laufenden Produktion genommen. Bis zur Analyse und zu den Umesterungsversuchen wurden die Proben unter Luft- und Lichtausschluß bei $+1^{\circ} \mathrm{C}$ gelagert. Tabelle 1 gibt einen Überblick über die Herkunft der Fette.

Tabelle 1: Herkunft der Altfettproben
\begin{tabular}{|l|l}
\hline Probenbezeichnung & Probenherkunft \\
\hline A & Mischfett nach Reinigung \\
\hline B & Mischfett nach Reinigung \\
\hline C & Mischfett nach Reinigung \\
\hline D & griechisches Restaurant \\
\hline E & deutsches Restaurant \\
\hline F & chinesisches Restaurant \\
\hline G & Schnellimbiss \\
\hline
\end{tabular}

\subsection{Analytik fettspezifischer Parameter}

Die Altfettproben wurden am Lehrstuhl für Energie- und Umwelttechnik der Lebensmittelindustrie auf Parameter untersucht, die insbesondere für die Auslegung des technischen Prozesses relevant sind. In Tabelle 2 sind die Prüfparameter mit den jeweiligen Standardmethoden gelistet.

Tabelle 2: Fettspezifische Parameter zur Charakterisierung der Altspeisefettproben

\begin{tabular}{l|l}
\hline Prüfparameter & Prüfverfahren \\
\hline Sulfatasche & DIN 51575 \\
\hline Gesamtverschmutzung & EN 12662 / DIN 51419-A \\
\hline Viskosität & DIN 51562 \\
\hline Jodzahl & DIN EN ISO 3961 \\
\hline Peroxidzahl & DGF Einheitsmethoden C-VI 6a (97) \\
\hline Säurezahl / freie Fettsäuren & DGF Einheitsmethoden C-V 2 (81) \\
\hline Wassergehalt & DIN 51777 / ASTM D 1744 \\
\hline Fettsäurespektren & EN ISO 5508 \\
\hline
\end{tabular}




\subsection{Spurenanalytik}

Dotierversuche. Die Altfettmethylester-Herstellung, deren Verlauf im Technikumsmaßstab in Abb. 2 dargestellt ist, wurde mit Altfett B (vgl. Tab. 1, 3-6, Abb. 1), jeweils dotiert mit verschiedenen Schadstoffen, im Labormaßstab durchgeführt. Anschließend wurden der Fettsäuremethylester, die zwei Glycerinphasen und die vereinigten Waschwässer getrennt analysiert, um den Verbleib der Spurenstoffe festzustellen.

Zur Durchführung der Umesterungsversuche wurden jeweils $25 \mathrm{~g}$ des Altspeisefettes vorgelegt und mit $500 \mu$ leines PCB Mischstandards (je $1 \mathrm{ng} / \mu \mathrm{l}$ von PCB $28\left(2,4,4^{\prime}\right.$-Trichlorbiphenyl), PCB 52 (2,2',5,5'-Tetrachlorbiphenyl), PCB 101

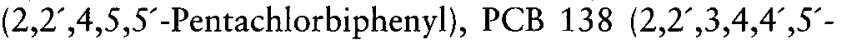
Hexachlorbiphenyl), PCB $153\left(2,2^{\prime}, 4,4^{\prime}, 5,5^{\prime}\right.$-Hexachlorbiphenyl) und PCB $180\left(2,2^{\prime}, 3,4,4^{\prime}, 5,5^{\prime}\right.$-Heptachlorbiphenyl)) versetzt. Ebenso wurden $250 \mu \mathrm{l}$ eines PAK-Mischstandards (1 ng/ $\mu$ l pro Einzelsubstanz, Auswahl von 15 PAK gemäß US-EPA, ohne Naphthalin) und $50 \mu \mathrm{l}$ eines PCDD/PCDF-

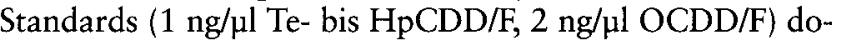
tiert. Das anfängliche Problem einer schlechten Phasentrennung nach Zugabe der Elemente $\mathrm{Al}, \mathrm{Cd}, \mathrm{Cr}, \mathrm{Cu}, \mathrm{Ni}, \mathrm{P}$, $\mathrm{Pb}, \mathrm{Sn}$ und $\mathrm{Zn}$ wurde durch die Erhöhung der Fetteinwaage auf $100 \mathrm{~g}$ behoben. Die Zugabe der Elemente erfolgte in Form von Ölstandards, in denen die Elemente komplexiert vorlagen (Specpure Metallo-Organic Standards, $1.000 \mu \mathrm{g} / \mathrm{g}$, Alfa Aesar / Johnson Matthey Company, Karlsruhe). In Zusatzversuchen wurden Oxide in fester Form eingebracht. Die Dotierungen wurden so vorgenommen, dass jeweils $1,0 \mathrm{mg}$ jedes Elementes dem Altspeisefett zugesetzt wurde.

Elementanalytik. Die Fettproben wurden einem mikrowelleninduzierten Druckaufschluss unterworfen (Aufschlussbedingungen: $0,5 \mathrm{~g}$ Probe, $8 \mathrm{ml} \mathrm{HNO}_{3}$ (65\%ig), $2 \mathrm{ml} \mathrm{H}_{2} \mathrm{O}_{2}(30 \% \mathrm{ig})$, $1 \mathrm{ml} \mathrm{H} \mathrm{O}_{2} \mathrm{O}$; stufenweise Erhitzung auf $200^{\circ} \mathrm{C}$, Abkühlung, Zugabe von $4 \mathrm{ml} \mathrm{H}_{2} \mathrm{O}_{2}$ und erneute Erhitzung auf $200^{\circ} \mathrm{C}$. Mikrowelle: Star-T-System, MLS GmbH). Die Elementgehaltbestimmungen erfolgten mittels optischer Emissionsspektrometrie mit induktiv gekoppeltem Plasma (ICP OES).

Analytik organischer Schadstoffe. PAK, PCB und PCDD/ PCDF wurden in den Matrizes Altspeisefett, Fettsäuremethylester und Glycerin wie in [6] beschrieben analysiert.
Die Waschwässer wurden jeweils mit dest. Wasser auf ein Volumen von $100 \mathrm{ml}$ aufgefüllt und wie nachfolgend beschrieben extrahiert:

PAK: Die wässrigen Proben wurden analog DIN 38407 Teil 8 zweimal mit je $20 \mathrm{ml}$ Cyclohexan extrahiert. Die vereinigten organischen Phasen wurden über $\mathrm{Na}_{2} \mathrm{SO}_{4}$ getrocknet und auf $1 \mathrm{ml}$ eingeengt.

PCB: Die Waschwässer wurden dreimal mit je $20 \mathrm{ml}$ Hexan extrahiert, die vereinigten organischen Lösungen dann über $\mathrm{Na}_{2} \mathrm{SO}_{4}$ getrocknet und auf $1 \mathrm{ml}$ eingedampft [7].

PCDD/F: Die wässrigen Phasen wurden mit $50 \mu$ eines ${ }^{13} \mathrm{C}-$ PCDD/F-Standards $(50 \mathrm{pg} / \mu \mathrm{l})$ versetzt und dreimal mit je $20 \mathrm{ml}$ Dichlormethan ausgeschüttelt [7]. Die vereinigten organischen Phasen wurden über $\mathrm{Na}_{2} \mathrm{SO}_{4}$ getrocknet und in $\mathrm{n}$ Heptan umgelöst. Die Lösungen wurden auf $2 \mathrm{ml}$ eingeengt.

Zur Analyse der organischen Schadstoffe in wässriger Matrix wurden nach der Extraktion Clean-up und Messungen ebenfalls gemäß [6] durchgeführt.

\section{Ergebnisse und Diskussion}

\subsection{Analysenergebnisse fettspezifischer Parameter}

In Tabelle 3 sind die Analysenergebnisse der fettspezifischen Parameter dargestellt. Abb. 1 zeigt die Fettsäurespektren der einzelnen Altspeisefettproben. Besonders interessant sind die Analysenergebnisse zu den Proben A, B und C, da es sich hierbei um gereinigte Mischfette handelt, wie sie am Markt erhältlich sind. Die Proben D bis G unterlagen, als Einzelproben aus verschiedenen Restaurants, schon aufgrund der Unterschiede in den eingesetzten Fetten relativ starken Eigenschaftsschwankungen.

Die Sulfataschegehalte der Altspeisefettproben lagen mit $0,001 \%-0,01 \%$ bei den Mischfettproben im Vergleich zu frischen unraffinierten Pflanzenölen relativ niedrig (Rapsöl 'Weihenstephaner Standard' max 0,01\%) [8]. Diese Unterschiede spiegelten sich in den Gesamtverschmutzungen GV nicht wider, die bei den Mischfettproben mit 2000 bis $4000 \mathrm{mg} / \mathrm{kg}$ sehr hoch waren (zum Vergleich: Rapsöl 'Weihenstephaner Standard' GV max. $25 \mathrm{mg} / \mathrm{kg}$ ). Durch die Umesterung mit

Tabelle 3: Analysenergebnisse der fettspezifischen Parameter

\begin{tabular}{l|c|c|c|c|c|c|c}
\hline Prüfparameter & A & B & C & D & E & F & G \\
\hline $\begin{array}{l}\text { Gesamtverschmutzung GV } \\
\text { [Massenanteil mg/kg] }\end{array}$ & 3.871 & 2.744 & 2.095 & 1.117 & 3.085 & 1.452 & 8.775 \\
\hline $\begin{array}{l}\text { Sulfatasche } \\
\text { [Massenanteil in \%] }\end{array}$ & 0,006 & 0,001 & 0,01 & 0,002 & 0,01 & 0,001 & 0,001 \\
\hline $\begin{array}{l}\text { kinematische Viskosität } \\
\text { [mm/s] }\end{array}$ & 47,5 & 46,3 & 47,0 & 42,4 & 43,6 & 44,0 & 43,4 \\
\hline $\begin{array}{l}\text { Wasser } \\
\text { [Massenanteil in \%] }\end{array}$ & 0,47 & 0,30 & 2,05 & 0,10 & 0,15 & 0,15 & 0,26 \\
\hline Peroxidzahl & 2,3 & 3,6 & 3,0 & 5,7 & 8,0 & 10,5 & 3,8 \\
\hline \begin{tabular}{l} 
lodzahl $[$ Gramm Jod / 100 g Fett] \\
\hline Säurezahl
\end{tabular} & 75 & 75 & 80 & 114 & 115 & 115 & 82 \\
\hline $\begin{array}{l}\text { freie Fettsäuren FFA } \\
\text { [Massenanteil in \%] }\end{array}$ & 2,66 & 2,46 & 6,80 & 0,60 & 0,55 & 0,80 & 2,10 \\
\hline
\end{tabular}




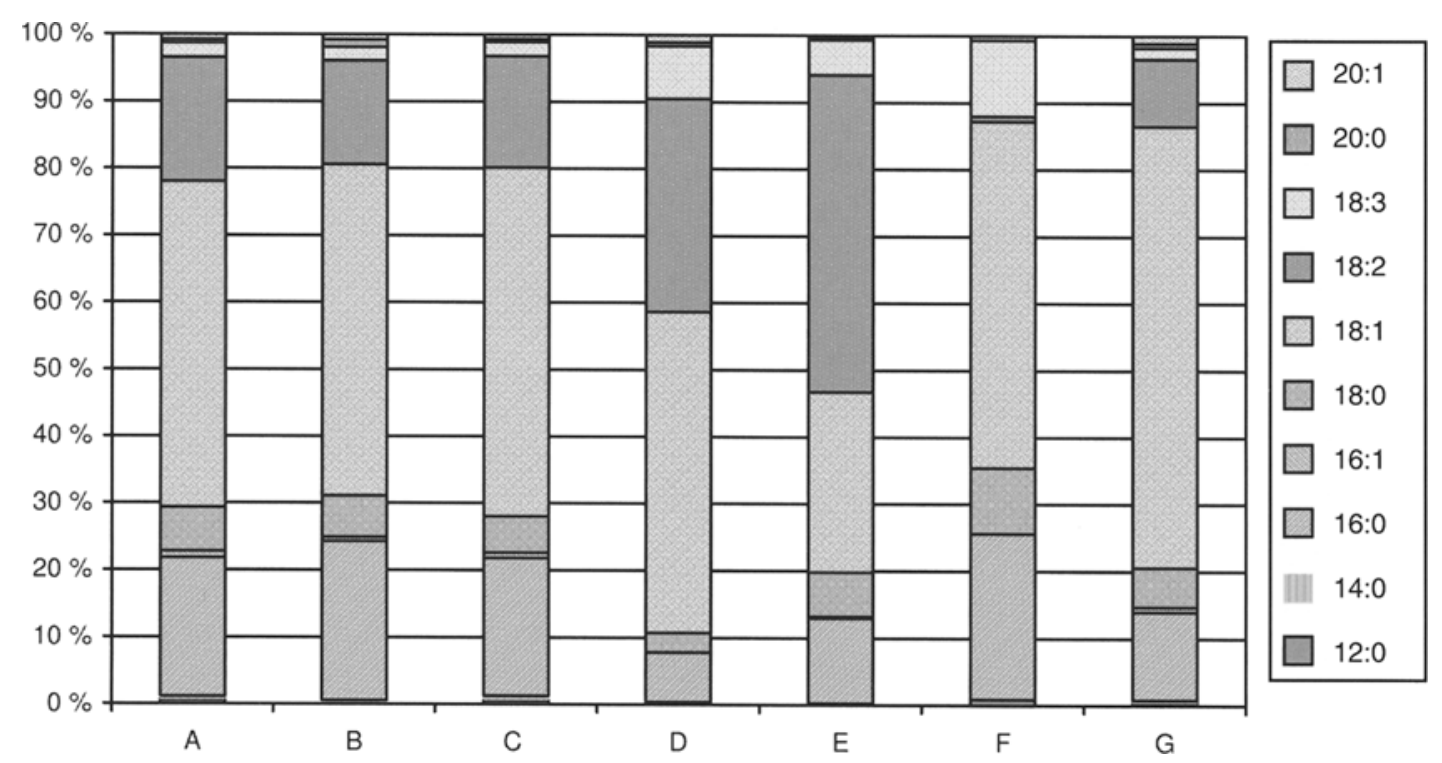

Abb. 1: Fettsäurespektren der Altspeisefettproben

Methanol bzw. mit anderen Alkoholen wird die Gesamtverschmutzung bei den Verfahrensschritten Glycerinabtrennung, Waschung und Destillation allerdings stark reduziert. Hinsichtlich der Viskosität wiesen die Fette nur geringe Unterschiede auf, die aus variierenden Fettsäurezusammensetzungen resultierten.

Die Iodzahlen der Mischfette waren erwartungsgemäß ähnlich. Bei den übrigen Fetten wurden in drei von vier Proben wesentlich höhere Werte gemessen. Vermutlich handelte es sich hier um gebrauchte ungehärtete Pflanzenöle (vgl. Fettsäurespektren). Die Peroxidzahlen lagen bei den Proben A bis $C$ in einem Bereich $<6$, so dass vom Vorliegen nicht oxidativ verdorbener Fette ausgegangen werden konnte (vgl. Matissek, R.: Lebensmittelanalytik, Springer 1992) [9]. Lediglich bei der Einzelfettprobe F wurde eine Peroxidzahl $>10$ gemessen, die eindeutig auf oxidativen Fettverderb schließen ließ.

Geringe Anteile an freien Fettsäuren wiesen auf einen niedrigen Grad des hydrolytischen Fettverderbs hin. Nur bei Probe $\mathrm{C}$ wurde ein hoher FFA Gehalt von fast $7 \%$ bestimmt, was auf starke Hydrolyse hindeutete. Dies wurde durch den hohen Wassergehalt von über $2 \%$ bestätigt. Vermutlich war diese Probe nach dem Reinigungsprozess mit Wasser verunreinigt worden, was während des Transports und der Lagerung zu starker Hydrolyse führte. Mit Ausnahme von Fett $C$ wiesen die Proben FFA Gehalte in einem Bereich auf, die sich kaum störend auf die Umesterungsreaktion auswirken. Der Katalysatorverlust durch die freien Fettsäuren ließ sich hier ohne Probleme durch leicht erhöhte Kat.-Menge ausgleichen, so dass bei Altspeisefetten guter Qualität ein einfaches basisch katalysiertes Umesterungsverfahren ohne saure Vorveresterung eingesetzt werden konnte. Die Wassergehalte lagen bei den Altfetten bis auf den Ausreisser C in einem niedrigen Bereich. Bei den Mischproben war vor der
Umesterung dennoch eine Wasserreduktion durch Destiliation angebracht, um den Katalysatorverlust zu verringern.

Die Fettsäurespektren der drei Mischfettproben waren nahezu identisch (Abb. 1). Sie wiesen einen gesättigten Anteil von ca. $30 \%$ auf und lagen somit unter dem Sättigungsgrad von Tierfetten. Auffällig war der hohe Anteil an Palmitinsäure und Linolsäure. Die Einzelfettproben zeigten deutliche Unterschiede in der Fettsäureverteilung.

Basierend auf diesen Analysenergebnissen wurde, in Anlehnung an bekannte Prozesse und basierend auf Erfahrungen des Lehrstuhls für Energie- und Umwelttechnik der Lebensmittelindustrie mit der Umesterung von Rapsöl, ein Verfahren zur basisch katalysierten Methylesterherstellung aus Altspeisefetten entwickelt. Als besonders geeignet schien ein batchweise arbeitendes zweistufiges Umesterungsverfahren zu sein, mit dem Biodiesel mit hoher Ausbeute und hervorragender Qualität aus kaltgepresstem Rapsöl hergestellt werden kann. Auf sauer katalysierte Vorveresterung konnte aufgrund des geringen Gehalts an freien Fettsäuren verzichtet werden. In Abb. 2 ist ein Fließdiagramm des angewandten Prozesses der Methylesterherstellung aus Altspeisefett mit den entsprechenden Massenströmen dargestellt. Dieses Verfahren gliedert sich in zwei durch $\mathrm{KOH}$ katalysierte Umesterungsschritte auf, einer sauren und einer neutralen Waschung und einer Destillation.

Beim ersten Umesterungsschritt wird ein Großteil des Altfettes zu Methylester umgesetzt. Durch die zweite Umesterung werden die noch zu ca. $5 \%$ vorhandenen Triglyceride größtenteils umgeestert. Auf diese Weise werden die von der Biodieselnorm DIN EN 14214 geforderten hohen Umesterungsgrade ohne Schwierigkeiten erreicht. Die saure Waschung dient dem Entfernen von Katalysatorresten. Durch die neutrale Waschung werden Säurereste und Salze entfernt. Die Destillation dient der Entfernung von Restmethanol und Wasser. 


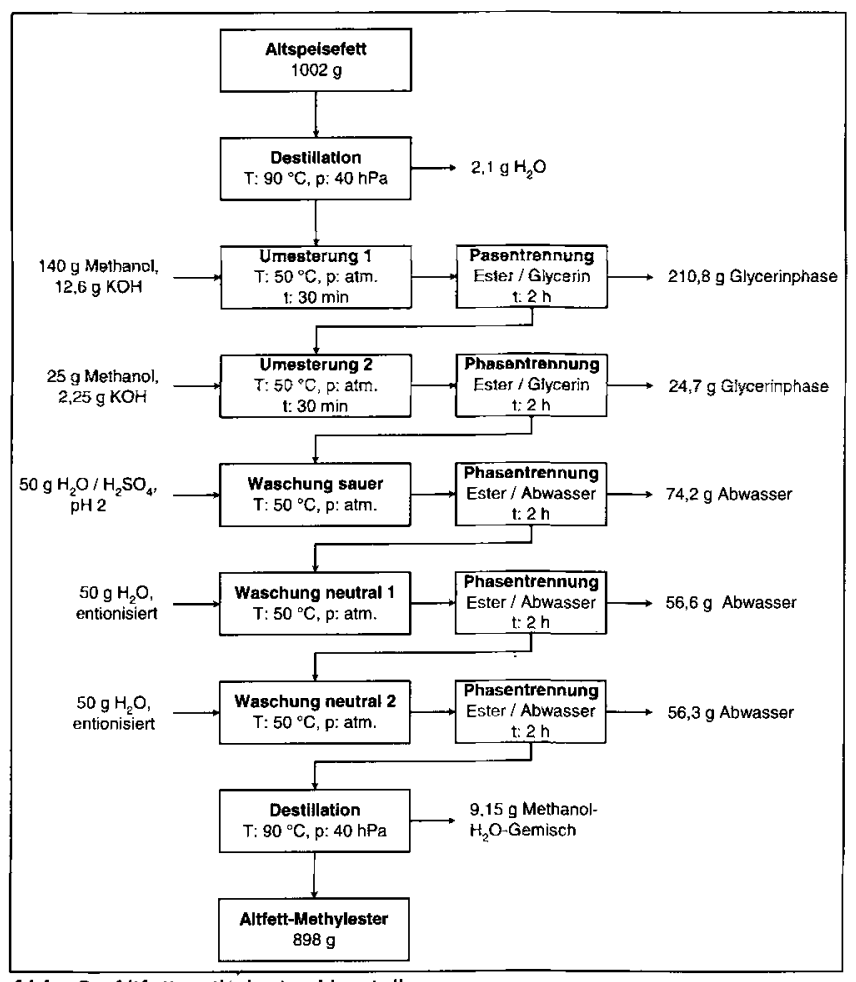

Abb. 2: Altfettmethylester-Herstellung

\subsection{Ergebnisse der Spurenanalytik}

Untersuchung eines Altspeisemischfettes. Da Mischfett B für die Dotierversuche eingesetzt werden sollte, wurden in dieser Matrix zunächst die Gehalte an PAK, PCB, PCDD/F sowie $\mathrm{Al}, \mathrm{Cd}, \mathrm{Cr}, \mathrm{Cu}, \mathrm{Ni}, \mathrm{P}, \mathrm{Pb}, \mathrm{Sn}$ und $\mathrm{Zn}$ bestimmt, die später zudotiert wurden. Die Ergebnisse dieser Analysen sind den Tabellen 4 bis 6 zu entnehmen.

PCDD und PCDF konnten nicht oberhalb der Bestimmungsgrenze von 0,1 ng Einzelkongener/g Fett nachgewiesen werden.

Hinsichtlich der Elementgehalte konnten offenbar typische Unterschiede zwischen dem hier untersuchten Altspeisemischfett und den technischen tierischen Fetten festgestellt werden, deren Eignung für die Kühlschmierstoff-Herstellung ebenfalls untersucht wird [6]. So waren einerseits, bedingt durch das Rohmaterial, in den Tierfetten die Phosphorkonzentrationen mit 84,6 bis $1.430 \mathrm{ng} \mathrm{P/g}$ Fett höher als die im Altspeisemischfett (vgl. Tab. 4). Andererseits wies das Altspeisefett einen höheren Zinngehalt als die Tierfette auf, was wahrscheinlich auf den Kontakt mit Konservendosen, d.h. Weissblech, zurückzuführen ist.

Das Altspeisefett weist hinsichtlich der PCB-Bełastung von $39,6 \mathrm{ng} / \mathrm{g}$ und des Kongenerenmusters eine hohe Übereinstim-

\begin{tabular}{c|c}
$\begin{array}{c}\text { Tabelle 4: Elementgehalte des Altspeisefettes 'Mischfett B nach Reinigung' } \\
\text { Element }\end{array}$ & Gehalt im Fett [ng/g Fett] \\
\hline $\mathrm{Al}$ & $<5$ \\
\hline $\mathrm{Cd}$ & $<2,5$ \\
\hline $\mathrm{Cr}$ & $<2,5$ \\
\hline $\mathrm{Cu}$ & $<2,5$ \\
\hline $\mathrm{Ni}$ & $<2,5$ \\
\hline $\mathrm{P}$ & 15,6 \\
\hline $\mathrm{Pb}$ & 3,01 \\
\hline $\mathrm{Sn}$ & 33,3 \\
\hline $\mathrm{Zn}$ & $<2,5$ \\
\hline
\end{tabular}

Tabelle 5: PCB-Gehalte des Altspeisefettes 'Mischfett B nach Reinigung'

\begin{tabular}{l|c}
\hline Substanz & Gehalt im Fett [ng/g Fett] \\
\hline PCB 28 & $<4$ \\
\hline PCB 52 & $<4$ \\
\hline PCB 101 & 7,03 \\
\hline PCB 138 & 10,6 \\
\hline PCB 153 & 13,7 \\
\hline PCB 180 & 8,26 \\
\hline Summe PCB & 39,6 \\
\hline
\end{tabular}

Tabelle 6: PAK-Gehalte des Altspeisefettes 'Mischfett B nach Reinigung'

\begin{tabular}{l|c}
\hline Substanz & Gehalt im Fett [ng/g Fett] \\
\hline Acenaphthylen & 2,29 \\
\hline Acenaphthen & 1,23 \\
\hline Fluoren & 3,58 \\
\hline Phenanthren & 16,9 \\
\hline Anthracen & 1,34 \\
\hline Fluoranthen & 5,54 \\
\hline Pyren & 7,02 \\
\hline Benz[a]anthracen & 1,40 \\
\hline Chrysen & 2,39 \\
\hline Benzo[b]fluoranthen & 1,43 \\
\hline Benzo[k]fluoranthen & 0,44 \\
\hline Benzo[a]pyren & 0,88 \\
\hline Indeno[1,2,3,c,d]pyren & 1,19 \\
\hline Dibenz[a,h]anthracen & 0,86 \\
\hline Benzo[g,h,i]perylen & 1,37 \\
\hline Summe PAK & $\mathbf{4 7 , 8}$
\end{tabular}

mung mit technischen tierischen Fetten auf [6]. Die Analysen dieser Fette ergaben PCB-Belastungen (Summe der sechs Leitsubstanzen) zwischen 30,2 und 74,1 ng/g Fett. Dabei fiel auf, dass die höher chlorierten Kongenere PCB 138, PCB 153 und PCB 180 in höheren Konzentrationen nachweisbar waren als die niedriger chlorierten (vgl. Tab. 5).

Wie im Fall der PCB wurde auch bezüglich der PAK-Analysenergebnisse eine auffällige Übereinstimmung zwischen dem Altspeisefett und den technischen tierischen Fetten festgestellt. Bei letzteren lagen die Konzentrationssummen der 15 untersuchten PAK Leitsubstanzen zwischen 25,0 und $121 \mathrm{ng} / \mathrm{g}$ Fett und Phenanthren war ebenfalls die dominierende Einzelsubstanz (vgl. Tab. 6).

Dotierversuche mit Elementen. Gemäß Abb. 2 fallen bei dem Umesterungsprozess, normiert auf die Einwaage des Altspeisefettes, 21,0\% Phase 1 Glycerin, 2,4\% Phase 2 Glycerin, $18,6 \%$ Waschwässer und $89,5 \%$ Fettsäuremethylester an. Auf diese also auch mengenmäßig unterschiedlichen Fraktionen verteilten sich während der Fettsäuremethylesterherstellung die Schadstoffe bzw. Elemente, die anfangs dem Altspeisefett zugesetzt worden waren.

Um den Umesterungsprozess nicht durch Zugabe saurer wässriger Lösungen zu stören und um realen Verhältnissen möglichst nahe zu kommen, wurden für die Dotierexperimente Elementstandards in Ölmatrix verwendet. Die resultierenden Verteilungsmuster sind in Abb. 3 dargestellt.

Es wurde festgestellt, dass das Leichtmetall $\mathrm{Al}$ während des Umesterungsprozesses ausschließlich in die Glycerinphasen übergeht. $\mathrm{P}$ und $\mathrm{Sn}$, beide in ihren Verbindungen toxikologisch eher unbedeutend, waren in Anteilen um $70 \% \mathrm{im}$ 


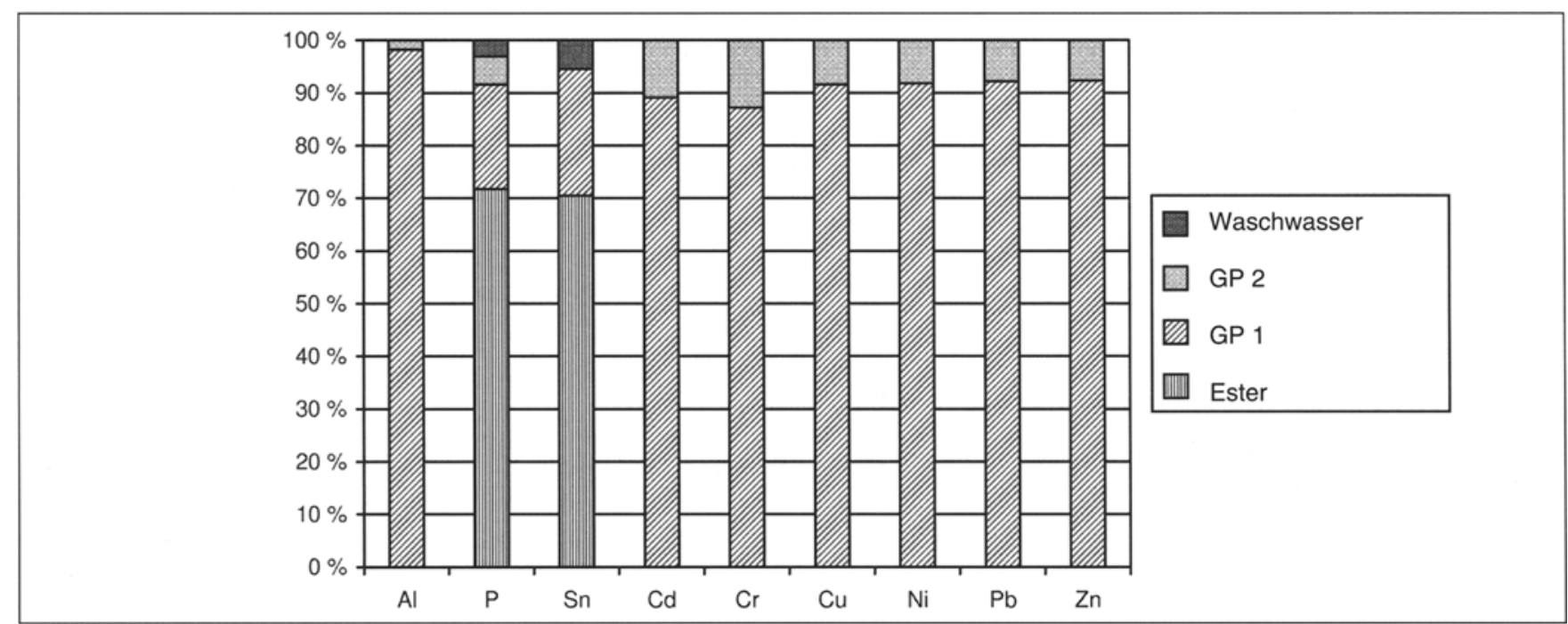

Abb. 3: Verteilung der Elemente nach Umesterung des Altspeisefettes

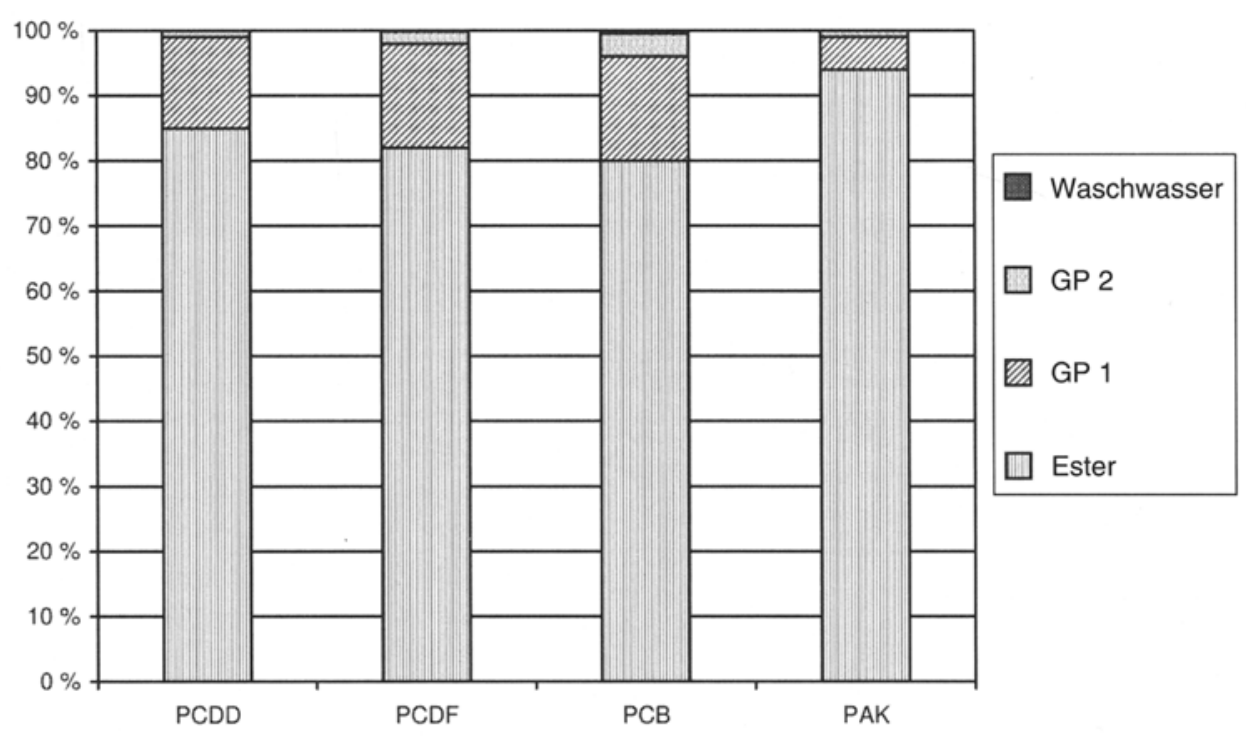

Abb. 4: Verteilung der organischen Schadstoffe nach Umesterung des Altspeisefettes

Fettsäuremethylester, zu rund 15 bis $20 \%$ in den Glycerinphasen aber auch in geringem Maße in den Waschwässern zu finden. Die Schwermetalle $\mathrm{Cd}, \mathrm{Cr}, \mathrm{Cu}, \mathrm{Ni}, \mathrm{Pb}$ und $\mathrm{Zn}$ reicherten sich recht einheitlich zu ca. $90 \%$ in der größeren Glycerinphase 1 und zu ca. 10\% im Phase 2 Glycerin an. $\mathrm{Zu}$ Vergleichszwecken wurden die Elemente pulverförmig als Oxide eingewogen und in dieser Form dem Altspeisefett vor der Umesterung zugesetzt. Tatsächlich konnten die o.g. Versuchsergebnisse trotz des veränderten experimentellen Ansatzes und einer etwas größeren Schwankungsbreite der Ergebnisse der Wiederholungsexperimente bestätigt werden.

Dotierversuche mit organischen Schadstoffen. In Abb. 4 werden die Ergebnisse der Dotierversuche mit den organischen Schadstoffen gezeigt.

Ein klarer Trend ist zu erkennen, der die Ähnlichkeit physikalischer Eigenschaften der eingesetzten schwerflüchtigen, lipophilen z.T. chlorierten Substanzen widerspiegelt. So wurden 80 bis $85 \%$ der PCDD, PCDF und PCB im Fettsäuremethylester ge- funden. 14 bis $16 \%$ bzw. 1 bis $4 \%$ befanden sich in den Glycerinphasen 1 und 2 und nur 0,1 bis $0,5 \%$ dieser Chlororganika konnten im organisch verunreinigten Waschwasser nachgewiesen werden. Die Gruppe der 15 PAK-Leitsubstanzen befand sich nach der Prozessdurchführung sogar zu $95 \%$ im Methylester, nur zu 5\% in den Glycerinphasen und konnte in den vereinigten Waschwässern nahezu nicht detektiert werden.

\subsection{Schlussfolgerung und Perspektive}

Die Analytik der fettspezifischen Parameter lieferte wertvolle Hinweise für die Auslegung des technologischen Prozesses der Methylesterherstellung aus Altspeisefett. So stellte sich zum Beispiel heraus, dass durch Entfernung des hohen Schmutz anteils und durch Entwässerung bessere Ergebnisse bei der Umesterung erreicht werden. Aufgrund der relativ niedrigen Gehalte an freien Fettsäuren ist eine saure Vorveresterung nicht zwingend nötig. Auch mit einem rein basenkatalysierten Prozess konnte eine Ausbeute an Fettsäuremethylestern von 
fast $90 \%$ erzielt werden. Der aus zweistufiger Umesterung, saurer und neutraler Waschung und Destillation aufgebaute Prozess ermöglicht die Einhaltung aller relevanten Grenzwerte der Blodieselnorm DIN EN 14214. Für die Weiterverwertung der Altfettmethylester als Rohstoff für die Herstellung von Kühlschmierstoffen empfiehlt sich allerdings noch eine Erhöhung des Anteils an gesättigten Fettsäuremethylestern zur Steigerung der Oxidationsstabilität.

Die spurenanalytische Untersuchung eines Altspeisemischfettes zeigte eine sehr niedrige Grundbelastung mit relevanten organischen Schadstoffen und Schwermetallen. Im Falle der Feststellung deutlich höherer Schadstoffkonzentrationen im Rohmaterial wäre sicherlich von einem unbeabsichtigten bis illegalen Fremdeintrag auszugehen. In diesem $\mathrm{Zu}-$ sammenhang belegen die Dotierexperimente zur Fettsäuremethylesterherstellung eine bevorzugte Anreicherung von Schwermetallen im Glycerin. Dieses Ergebnis ist von Bedeutung, da später auch das während der Esterproduktion anfallende Rohglycerin ein verkaufsfähiges Handelsprodukt darstellt. Für die hier untersuchten schwerflüchtigen organischen Schadstoffe wurde ein gänzlich anderes Verteilungsmuster bestimmt. Sie wurden zu 80 bis $95 \%$ im Esterprodukt wiedergefunden, was je nach Auslegung des weiteren Prozesses zur Herstellung der KSS-Grundöle durchaus zu deren Kontamination führen könnte. Die Waschwässer, deren Aufreinigung ebenfalls $z u$ berücksichtigen ist, wurden von keinem der Schadstoffe nennenswert kontaminiert.

Der Prozess der Weiterverarbeitung der Methylester zu Monoalkylestern mit Alkoholen der Kettenlängen C2 bis C8 zwecks Einstellung der benötigten Viskositäten der Basisöle wird in einem nächsten Schritt vorgestellt werden. Damit verknüpft werden Ergebnisse chemisch-analytischer Unter- suchungen, die sowohl beim Herstellungsprozess als auch während des KSS-Einsatzes an der Werkzeugmaschine im Versuchsbetrieb wie in der Serienfertigung durchzuführen sind, veröffentlicht werden.

\section{Literatur}

[1] Süß AAA (1999): Wiederverwertung von gebrauchten Speiseölen/-fetten im energetisch technischen Bereich. VDI Fortschritt-Berichte Reihe 15 Nr. 219

[2] Falk O, Sutor G, Wiegland S (2001): Altspeisefette: Aufkommen und Verwertung. Studie 2001, Internetangebor des Lehrstuhls für Energieund Umwelttechnik der Lebensmittelindustrie $<$ http://www.wzw.tum. de/blm/eul/s

[3] Jurisch C, Meyer-Pittroff R (1995): Verwertung von pflanzlichen und tierischen Altölen und -fetten zur Herstellung regenerativen Kraftstoffs. Schlussbericht CARMEN e.V., Technische Universität München

[4] Kersting R, van der Pütten N (1996): Entsorgung von Altfetten in Hessen: Situation, Handlungsbedarf. Hessische Landesanstalt für Umwelt. Heft 222, Wiesbaden

[5] Neumann C (1999): Wie die Laborratte ins Schweineschnitzel kommt. Süddeutsche Zeitung vom 17.06.99, S, L12

[6] Bahadir M, Bock R, Dettmer T, Falk O, Hesselbach J, Jopke P, Matthies B, Meyer-Pittroff R, Schmidt-Naedler C, Wichmann H (2004): Chemisch-analytische Charakterisierung technischen tierischen Fettes aus einer Tierkörperbeseitigungsanstalt. UWSF $-\mathrm{Z}$ Umweltchem Ökotox 16 (1) $19-28$

[7] Rump HH, Scholz B (1995): Untersuchung von Abfällen, Reststoffen und Altlasten. VCH Verlagsgesellschaft mbH, Weinheim

[8] Remmele E, Thuneke T, Widmann B, Wilharm T, Schön H (2000): Begleitforschung zur Standardisierung von Rapsöl als Kraftstoff für pflanzenöltaugliche Dieselmotoren in Fahrzeugen und BHKW. 'Gelbes Heft' Nr. 69. Hrsg. und Druck: Bayerisches Staatsministerium für Enährung, Landwirtschaft und Forsten, München

[9] Matissek R, Schnepel, Steiner G (1992): Lebensmittelanalytik. Springer Verlag Berlin, 2. Auflage

Eingegangen: 12. September 2003 Akzeptient: 06. Februar 2004 OnlineFirst: 09. Februar 2004

\section{Biokatalytisches Verfahren zur Herstellung von Fettsäureestern (Esteröle)}

Von der Deutschen Bundesstiftung Umwelt (DBU) wird im Rahmen der Initiative 'InnovationsCentrum Biokatalyse' das Vorhaben 'Enzymatische Altfettalkoholyse zur Herstellung von Wertstoffen' (Az 13078) gefördert. Mit Hilfe von Enzymen ist es möglich, im Temperaturbereich 50 bis $70^{\circ} \mathrm{C}$ aus (Alt-)Fetten und nichtwassermischbaren Alkoholen Fettsäureester (Esteröle) zu erzeugen, die im Schmiermittelsektor ihre Anwendung finden sollen. Der enzymatische Prozess ist bisher bis in den $\mathrm{t}-\mathrm{Maßstab}$ übertragen worden. Parallel zu technologischen Untersuchungen wird der gesamte Lebensweg des Produkts ökobilanziert. Zur Zeit wird nach erfolgreichen Laborversuchen der Einsatz eines Esters als Kühlschmierstoff in der Serienferti- gung bei der Volkswagen AG, Werk Salzgitter, vorbereitet. Projektpartner sind: Bundesanstalt für Materialforschung und -prüfung (BAM) / Berlin, Institut für Werkzeugmaschinen und Fertigungstechnik (IWF) der TU Braunschweig, Volkswagen AG Wolfsburg, Castrol GmbH Mönchengladbach und Greibo-Chemie Velten.

Chemisch-analytische Untersuchungen werden vom Instilut für Ökologische Chemie und Abfallanalytik der Technischen Universität Braunschweig durchgeführt.

Korrespondenzadresse: rudolf.brenneis@bam.de 